\title{
On the resolution limit of digital particle image velocimetry
}

\author{
Christian J. Kähler · Sven Scharnowski • \\ Christian Cierpka
}

Received: 30 September 2011 /Revised: 19 January 2012/ Accepted: 6 February 2012/Published online: 21 February 2012

(C) The Author(s) 2012. This article is published with open access at Springerlink.com

\begin{abstract}
This work analyzes the spatial resolution that can be achieved by digital particle image velocimetry (DPIV) as a function of the tracer particles and the imaging and recording system. As the in-plane resolution for window-correlation evaluation is related by the interrogation window size, it was assumed in the past that single-pixel ensemble-correlation increases the spatial resolution up to the pixel limit. However, it is shown that the determining factor limiting the resolution of single-pixel ensemblecorrelation are the size of the particle images, which is dependent on the size of the particles, the magnification, the f-number of the imaging system, and the optical aberrations. Furthermore, since the minimum detectable particle image size is determined by the pixel size of the camera sensor in DPIV, this quantity is also considered in this analysis. It is shown that the optimal magnification that results in the best possible spatial resolution can be estimated from the particle size, the lens properties, and the pixel size of the camera. Thus, the information provided in this paper allows for the optimization of the camera and objective lens choices as well as the working distance for a given setup. Furthermore, the possibility of increasing the spatial resolution by means of particle tracking velocimetry (PTV) is discussed in detail. It is shown that this technique allows to increase the spatial resolution to the subpixel limit for averaged flow fields. In addition, PTV evaluation methods do not show bias errors that are typical for correlation-based approaches. Therefore, this technique is best suited for the estimation of velocity profiles.
\end{abstract}

C. J. Kähler $(\bowtie) \cdot$ S. Scharnowski · C. Cierpka Institute of Fluid Mechanics and Aerodynamics, Universität der Bundeswehr München,

85577 Neubiberg, Germany

e-mail: Christian.Kaehler@unibw.de

\section{Introduction}

Digital particle image velocimetry (DPIV) is a non-intrusive measurement technique that estimates the velocity field of a flow in a plane, or even in a volume, by measuring the displacement of appropriate particles or particle groups in a certain time interval $\Delta t$. Therefore, tracer particles that follow the fluid motion faithfully are illuminated twice by a laser and the light scattered from the particles at $t$ and $t+\Delta t$ is recorded on successive frames of a digital camera. In a second step, the recorded image pair is subdivided into several thousand interrogation windows, and the average particle image displacement is estimated for each interrogation window by using spatial correlation methods with iterative multi-grid and image deformation techniques. Finally, the local flow velocity for each interrogation window is estimated from the location of the signal peak in the correlation plane by taking into account the optical magnification of the imaging system and the time interval between the two illuminations (Adrian and Westerweel 2010; Raffel et al. 2007). Due to the recording principle, each measured velocity vector represents a volume-averaged mean motion of the discretized and quantized tracer particle's diffraction images, rather than the actual velocity of the flow at $\mathbf{r}$. This can be expressed by the following equation:

$\langle\mathbf{u}(\mathbf{r}, t)\rangle=\int_{\Delta V} G\left(\mathbf{r}, \mathbf{r}^{\prime}, S\right) \cdot \mathbf{u}\left(\mathbf{r}^{\prime}, t\right) d V^{\prime}$.

The weighting function $G\left(\mathbf{r}, \mathbf{r}^{\prime}, S\right)$ accounts for the intensity and location $\mathbf{r}^{\prime}$ of the particle images as well as the discrete sampling due to the digital recording. This weighting function strongly depends on the illumination and imaging system, including the laser beam profile, the 
camera pixel size $S$, the fill factor of the camera sensor, and the modular transfer function (MTF) of the objective lens and the microlens array above the CCD/CMOS sensor. The measurement volume $\Delta V$ is mainly determined by the interrogation window size projected in physical space, the light sheet thickness, and the particle size, because small particles at the border of the light sheet may not scatter enough light for digital registration.

Generally, double pulse DPIV is not capable of detecting the path and acceleration of the tracer particles, as illustrated in Fig. 1. This would require multi-pulse DPIV systems as outlined in Kähler and Kompenhans (2000) in combination with multi-frame evaluation techniques as discussed in detail in Hain and Kähler (2007). Therefore, the higher order effects caused by acceleration and curvature must be avoided in double pulse DPIV by selecting a sufficiently short time separation $\Delta t$ between the laser pulses and a sufficiently large magnification $M$ of the imaging system such that the displacement can be resolved properly. In this case, the first-order approximation of the particle path, indicated by the straight line in Fig. 1, matches well with the true particle motion within the uncertainty of the method.

In the case of supersonic flows with shocks located between the two particle positions, as illustrated in Fig. 2, additional attention is required. Although the straight line between the particle positions may be the actual path of

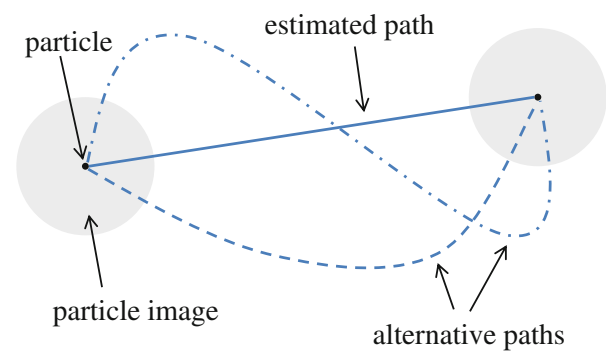

Fig. 1 Three hypothetical trajectories of a particle (black dots) and its images (gray circles) at the beginning and the end of the motion



Fig. 2 Effect of strong acceleration due to a shock between the particle image pair in a supersonic flow motion, the measured velocity estimated from the two particle locations and the time interval $\Delta t$ depends on the exact shock location with respect to the particle positions. The particle lag is not considered here as this bias error also appears for molecular tagging methods, which do not show any lag at all.

Besides these local effects related to individual particles, which are also relevant for particle tracking and molecular tagging techniques, DPIV suffers from averaging the motion of several particle images within the interrogation windows. This causes significant bias errors in case of flow gradients as illustrated in Fig. 3 (Keane and Adrian 1990; Westerweel 2008). In order to minimize the errors associated with spatial averaging, it is essential, first of all, to acquire proper data with a well-aligned system. Secondly, the time interval between the illuminations must be sufficiently short to overcome the problems discussed above. Finally, since this increases the relative measurement uncertainty as the particle image displacement becomes smaller, while the precision in estimating the signal peak in the correlation plane is constant, the spatial resolution must be increased to maintain precision for the velocity estimation and to sample the flow gradients correctly.

To increase the spatial resolution of DPIV systems, as well as its measurement precision, long-range micro-PIV presents a well-established method when the observation distance cannot be further reduced or when perspective errors are essential and must be avoided, as outlined in Kähler et al. (2006). This technique, used in combination with single-pixel evaluation, increases the resolution significantly. Single-pixel ensemble-correlation evaluates a large number of PIV images, which are divided in two sets. The first set contains all the first images of each image pair, and the second set contains the corresponding second images that were acquired at $\Delta t$ after the first one. The double images do not need to be captured at equidistant time steps, but the interval between all image pairs $\Delta t$ does need to remain constant. The time plot for the image intensity at a certain pixel from the first image set is

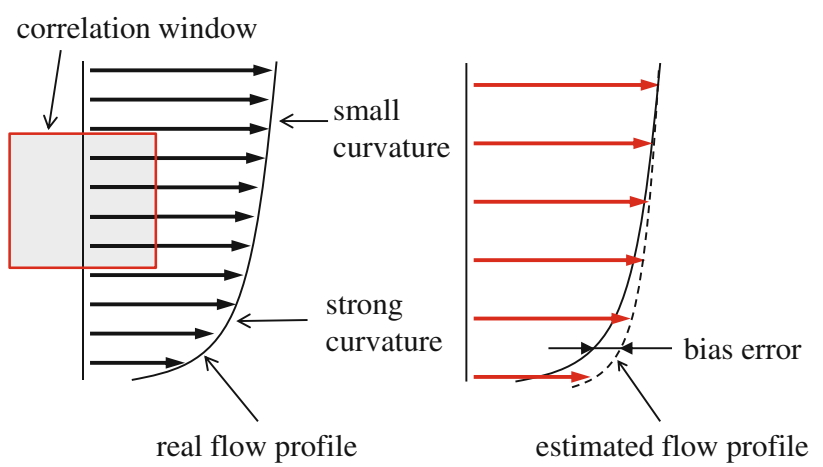

Fig. 3 Spatial filtering of velocity profiles caused by windowcorrelation 
correlated with the time plot of neighboring pixels in the second set, and a correlation map is generated. The position of the peak of this correlation map corresponds to the tracer particle (ensemble averaged) mean displacement, and the shape of the correlation yields the information about the average fluctuations. Single-pixel ensemble-correlation was first applied by Westerweel et al. (2004) for stationary laminar flows in microfluidics. In the last years, the approach was extended for the analysis of periodic laminar flows (Billy et al. 2004), of macroscopic laminar, transitional, and turbulent flows (Kähler et al. 2006), and for compressible flows at large Mach numbers (Kähler and Scholz 2006; Bitter et al. 2011). Scholz and Kähler (2006) have extended the high-resolution evaluation concept for stereoscopic PIV recording configurations and recently, the single-pixel evaluation was further developed to estimate Reynolds stresses in turbulent flows (Kähler and Scholz 2006; Scharnowski et al. 2011).

However, although it is possible to compute velocity vectors even in smaller scales than a micrometer grid, these vectors are not independent and bias errors can occur in particular situations. Even though many different approaches to increase the accuracy and resolution of DPIV were presented, see Adrian and Westerweel (2010), Keane et al. (1995), Raffel et al. (2007), Scarano (2001), Stanislas et al. (2003, 2005, 2008), Stitou and Riethmuller (2001), Willert (1997), for instance, a detailed analysis of the resolution limit is still lacking and will be the focus of this paper.

Section 2 discusses how a step function is used to determine the spatial resolution of DPIV evaluation methods. In Sect. 3, the effect of the size of the particle images as a function of the optical magnification is analyzed in detail, and in Sect. 4, the response to a step-like velocity profile is analyzed for window-correlation (Sect. 4.1), ensemble-correlation (Sect. 4.2), and particle tracking velocimetry (Sect. 4.3), respectively, in order to identify the dependence of the resolution on the particle image size. Finally, the spatial resolution and the optical magnification are linked together in Sect. 5.

\section{Step function response}

To determine the effective resolution for various interrogation approaches systematically, the resolution limit can be analyzed with a step-like velocity profile, represented by a contact discontinuity layer in supersonic flows, for instance:

$\Delta X^{*}\left(Y^{*}\right)=\left\{\begin{array}{ll}1 \mathrm{px} & Y^{*} \geq 0 \\ 0 \mathrm{px} & Y^{*}<0\end{array}\right.$.

The step response is also frequently used in electrical engineering and control theory to analyze an output's behavior when the input signal changes in a very short time. In order to analyze the DPIV resolution, the signal (particle image displacement) is changed in space over a very short distance. The response to a step profile is shown in the top profile of Fig. 4 for window-correlation using four different interrogation window sizes and single-pixel ensemble-correlation.

The width of the response function (step response width or SRW) can be regarded as the resolution and describes the minimum distance between independent vectors. Only for distances larger than the SRW, the vector is not biased by the aforementioned flow variations.

Window-correlation leads to a broad response that is dependent on the interrogation window size. This becomes even more evident when gradient-based quantities are considered, as shown in the lower part of Fig. 4. The gradients are underestimated, and their spatial extension is enlarged. It is obvious that this effect leads to systematic errors in estimating the vorticity and other gradient-based quantities such as dissipation for instance. The use of weighting functions for the strongly overlapping interrogations windows can improve the spatial resolution to a certain extend. However, independent vectors can be determined with a resolution of about $4 . .5$ pixels only under ideal conditions as shown by Nogueira et al. (2005).
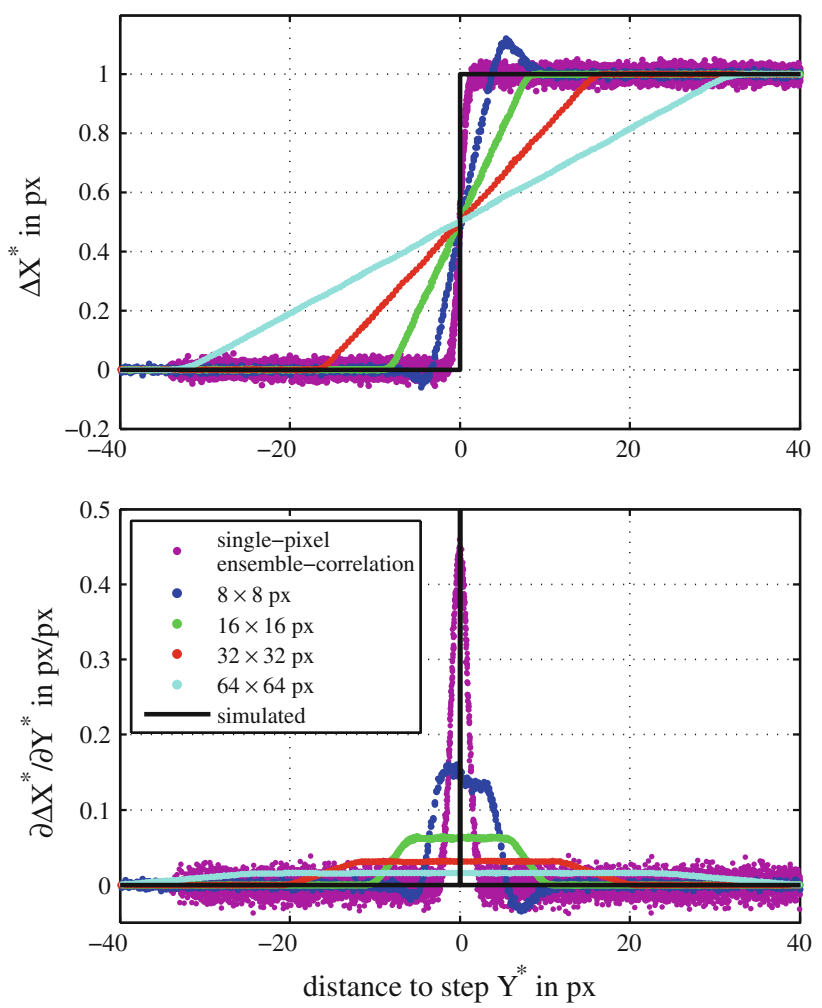

Fig. 4 Response to a step-like velocity profile (top) and the corresponding velocity gradients (bottom) computed with different interrogation window sizes and with single-pixel ensemble-correlation. A digital particle image diameter of $D=3 \mathrm{px}$ was used for the synthetic images 
Single-pixel ensemble-correlation can drastically reduce the spatial filtering and is therefore well suited to increase the spatial resolution. However, it should be noted that it does still not represent the exact solution. The deviation is due to the fact that the measured mean velocity represents the convolution of the particle image (or more precisely, of the particle images' auto-correlation) and the actual velocity distribution as outlined in Scharnowski et al. (2011). Therefore, the question arises: How can the resolution be increased or what is the best spatial resolution that can be achieved by DPIV?

\section{Large particle images and their implications}

As mentioned above, by increasing the magnification, the resolution of the measurements can be enhanced. However, the imaging of the particles is strongly affected by the optical magnification. The particle image diameter on the image plane is directly related to the particle size itself via the optical magnification. However, it appears enlarged in the DPIV images mainly due to four effects:

1. diffraction at the limited aperture of the objective lens

2. defocussing

3. lens aberrations

4. discretization and quantization of the continuous image signal into a discrete signal with pixel size $S$.

The enlargement of the particle image due to diffraction and defocussing can be described by the second and third term under the square root of the following equation determined by Olsen and Adrian (2000):

$d_{\tau}=\sqrt{\left(M \cdot d_{p}\right)^{2}+\left(2.44 \cdot f_{\#}(M+1) \lambda\right)^{2}+\left(\frac{M \cdot z \cdot D_{a}}{s_{0}+z}\right)^{2}}$

where $M$ is the magnification of the imaging system, $d_{\mathrm{p}}$ the particle diameter, $f_{\#}$ the ratio between objective lens diameter and the aperture's diameter, $\lambda$ the wavelength of the scattered light (or the fluorescent light in microfluidics), $z$ the object's distance from the focal plane, $D_{\mathrm{a}}$ the lens aperture diameter, and $s_{0}$ the object distance. Frequently used variables are also summarized in Table 1.

The three terms in the square root of Eq. 3 correspond to the geometric, diffraction, and defocussing components. The latter shows only significant influence for volume illumination, typically used in micro-PIV (Rossi et al. 2011). In macroscopic DPIV, $z$ is usually $1-3$ orders of magnitude smaller than $s_{0}$ for well-aligned optical systems. In the case of low magnification, the diffraction-limited particle image (on the image plane) is smallest and has the lower limit of $2.44 \cdot f_{\#} \cdot \lambda$ for $M \rightarrow 0$. Thus, for a large
Table 1 Frequently used variables and their meaning

\begin{tabular}{lll}
\hline Quantity & Symbol & Unit \\
\hline Particle diameter & $d_{\mathrm{p}}$ & $\mu \mathrm{m}$ \\
Particle image diameter & $d_{\tau}$ & $\mu \mathrm{m}$ \\
Digital particle image diameter & $D$ & $\mathrm{px}$ \\
Dynamic spatial range & DSR & $\mathrm{m} / \mathrm{m}$ \\
Field of view & FOV & $\mathrm{m}$ \\
F-number & $f_{\#}$ & $\mathrm{~m} / \mathrm{m}$ \\
Discrete sensor size & $L$ & $\mathrm{px}$ \\
Optical magnification & $M$ & $\mathrm{~m} / \mathrm{m}$ \\
Sensor pixel size & $S$ & $\mu \mathrm{m} / \mathrm{px}$ \\
Spatial resolution & res & $\mathrm{m}$ \\
Step response width & SRW & $\mathrm{px}$ \\
Shift vector components & $\Delta X, \Delta Y$ & $\mathrm{px}$ \\
\hline
\end{tabular}

numerical aperture (small $f_{\#}$ ) and a wavelength in the range of visible light, the particle images are smaller than the pixel size $S$ of the camera sensor, which is typically in the range of 5-20 $\mu \mathrm{m}$ for typical CCD or CMOS cameras used for DPIV, according to Hain et al. (2007).

However, after the discretization and quantization of the image by means of the discrete pixels of the digital camera sensor, all particle images will have a size of at least 1 pixel. Thus, smaller particle images are artificially enlarged in the case of low magnification. The digital particle image diameter $D$ on the measurement plane is dependent on the pixel size $S$ and the particle image diameter $d_{\tau}$. For particle images $d_{\tau}<S$, the digital particle image diameter $D$ is equal to 1 pixel or slightly larger. Even a very small particle image can illuminate up to 4 pixel if it is located close to the pixel's corner. However, the probability that a small particle image illuminates only one single pixel on the sensor is $\left(S-d_{\tau}\right)^{2} / S^{2}$. For particle images larger than 1 pixel $\left(d_{\tau}>S\right)$, the following expression holds:

$D \approx d_{\tau} / S$

Figure 5 illustrates the effective particle image diameter as a function of the optical magnification for $f_{\#}=2$ and $d_{\mathrm{p}}=1 \mu \mathrm{m}$, which is a typical particle diameter for air flow, according to Wernet and Wernet (1994), Melling (1997), and Kähler et al. (2002). Additionally, a horizontal line representing the pixel size is drawn at $S=9 \mu \mathrm{m}$, which corresponds to the PCO.4000 camera, for reference purposes. Only particle image diameters above both curves can possibly exist in reality for this setup. Besides, the theoretical functions are plotted along with experimental results, where the particle image diameter was measured at $1 / e^{2}$ of the peak intensity. The experimental results were acquired by using a Makro-Planar $T \times 2 / 100$ objective lens (by Carl Zeiss AG) combined with up to six tele-converters ( $2 x$ Pro 300 by Kenko Tokina Co., Ltd.) connected to a 


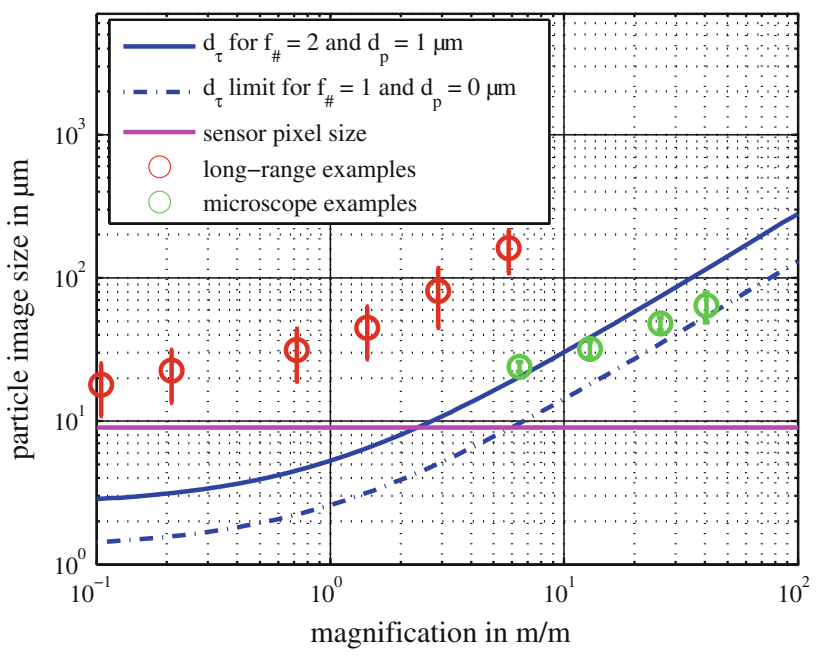

Fig. 5 Limitation of the particle image size due to diffraction, aberrations, and discretization

PCO.4000 camera (by PCO AG). The working distance was set to approximately $1 \mathrm{~m}$, and the results are referred to as 'long-range' results. Additionally, the particle image diameters of polystyrene latex particles $\left(d_{\mathrm{P}}=1 \mu \mathrm{m}\right)$, fabricated by microparticles $\mathrm{GmbH}$ and coated with a red fluorescent dye, were analyzed. The particles were observed using a Zeiss Axio Observer.Z1 inverted microscope coupled with a double-cavity frequencydoubled Litron Nano S Nd:YAG laser $(532 \mathrm{~nm})$ for the illumination of the particles. The particle images were recorded with a PCO Sensicam QE camera. The results are shown for different magnifications in Fig. 5. Some example images are illustrated in Fig. 6.

The difference between the experimental long-range results and the theory is mainly due to lens aberrations,
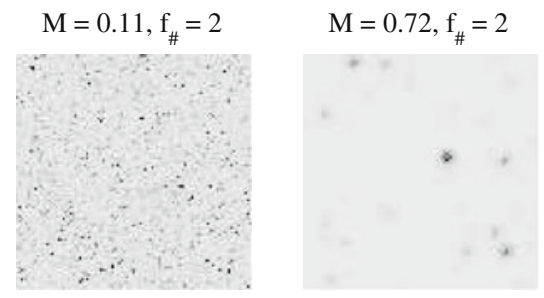

$\mathrm{M}=6.45, \mathrm{f}_{\#}=1.67$
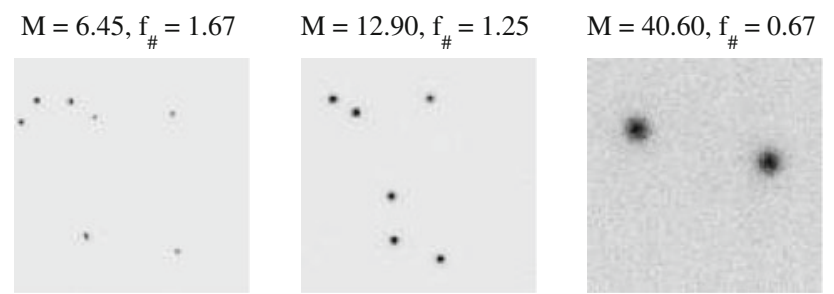

Fig. 6 Experimental digital particle images captured with different magnifications for the long-range example with a working distance of $1 \mathrm{~m}$ (top) and for the microscopic example (bottom) from Fig. 5. All images show a sample of $100 \times 100 \mathrm{px}$ since the MTF is fairly low for the high spatial frequencies caused by the small tracer particles and the large distance. Additionally, according to Eq. 3, slightly imperfect focusing leads to further broadening of the particle images. However, it is clearly visible in Fig. 5 that all the recorded particle images at $f_{\#}=2$ are larger than what the theoretical functions predict, as expected. Thus, these functions represent the theoretical lower limit for perfect (aberration free) imaging. For the particle imaging with the inverted microscope, for each magnification: $M=[6.45 ; 12.9 ; 25.5$; $40.6]$, the $f$-number changes to $f_{\#}=[1.67 ; 1.25 ; 0.83 ; 0.67]$. The working distance is in the millimeter range; thus, the MTF is quite large. Consequently, the particle image diameter is close to the theoretical limit, but still biased by optical aberrations.

\section{Resolution limit}

The response to a step-like velocity profile (as shown in Fig. 4) is well suited to determine the spatial resolution of the estimated velocity field. Such a strong local change does exist in reality in the form of contact layers in supersonic flows or at interfaces between different fluids (liquid-gas interface, for instance). Velocity distributions in high-speed shear layers and phase boundaries of two-phase flows, for instance, also represent a step function as particles are statistically not present directly at the interface.

\subsection{Window-correlation: resolution limit}

In the case of window-correlation, 1,000 synthetic image pairs of the size $256 \times 256$ px were generated for each particle image diameter considered in this analysis. The images were divided in two parts: one with zero velocity and one with a particle image motion of 1 pixel. The intersection line was slightly tilted $(1: 20)$ with respect to the image grid (pixel graticule), and the shift was applied parallel to this line to produce results for random subpixel locations. The seeding concentration was $25 \%$ in each case, meaning that $25 \%$ of the image area was covered by particle images. Consequently, the number of particles changes with the particle image diameter (as it does in real PIV recordings for magnified images and constant seeding concentration). Gaussian particle images were used before discretization. To reproduce the effect due to the finite pixel size of the camera sensor as close as possible, each pixel's gray value is computed from the integral of the intensity over the corresponding area, instead of simply transferring the analytical point-wise values to the pixel. This integral represents an idealized camera sensor with a fill factor of one and a constant transfer function. In reality, the transfer function may not be constant due to 
$\mathrm{D}=1 \mathrm{px}$



$\mathrm{D}=10 \mathrm{px}$



$\mathrm{D}=3 \mathrm{px}$

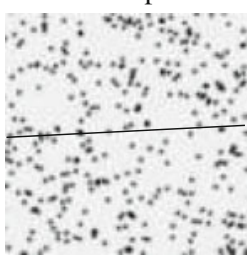

$\mathrm{D}=15 \mathrm{px}$

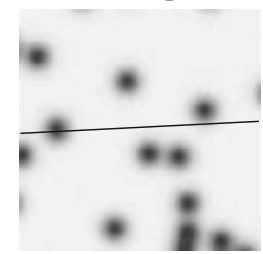

$\mathrm{D}=5 \mathrm{px}$
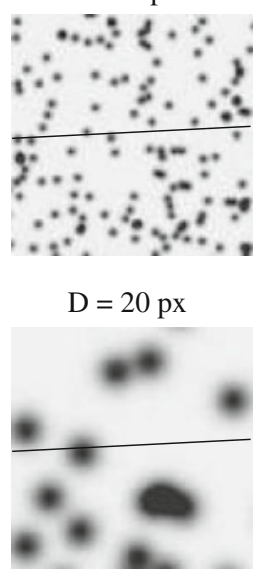

$\mathrm{D}=20 \mathrm{px}$

Fig. 7 Synthetic images for the step response test with different digital particle image diameters $D$. The intersection between the two velocity regions is indicated by the black solid line. All images show a sample of $100 \times 100 \mathrm{px}$

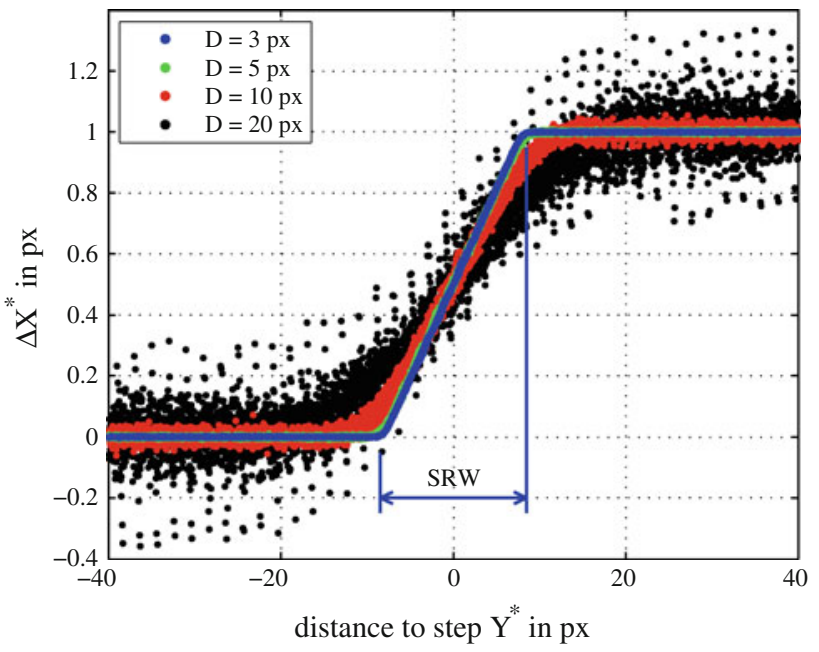

Fig. 8 Response to a step-like velocity profile for different digital particle image sizes computed with window-correlation

microlenses and the design of the sensor, as discussed in Kähler (2004, Section 2.3). However, for the present analysis, this simplification is irrelevant. The maximum intensity of the particle images was set to 1,000 counts, whereas in case of overlapping particle images, the intensity is the sum of the gray levels. A signal-to-noise ratio of SNR $=100: 1$ was simulated by means of a background noise with Gaussian distribution with a zero mean intensity and a standard deviation of 0.01 of the maximum intensity. Some example images are shown in Fig. 7.

The evaluation of the data was performed using a sumof-correlation approach proposed by Meinhart et al. (2000) without any window weighting using $16 \times 16 \mathrm{px}$ interrogation windows with $87 \%$ overlap. Figure 8 shows the displacement $\Delta X^{*}$ parallel to the intersection line with respect to the distance $Y^{*}$ from this line. It is clearly visible that the SRW depends strongly on the interrogation window size, as already illustrated in Fig. 4, but interestingly also on the size of the particle images as can be seen in Fig. 8. The response function becomes broadened (and also more noisy) for larger particle images. Hence, sum-ofcorrelation evaluation allows for reducing the interrogation window size down to approximately two times the particle image diameter. It is important to note that even the use of smaller interrogation windows does not gain any additional spatial resolution.

\subsection{Single-pixel ensemble-correlation: resolution limit}

In the case of single-pixel evaluation, more images are required (Scharnowski et al. 2011; Westerweel et al. 2004), and thus, 10,000 image pairs were generated for each particle image diameter considered in this analysis. For each pixel, the correlation function was computed using the approach outlined by Westerweel et al. (2004). The mean shift vector was estimated with a 3-point Gaussfit. Figure 9 shows the resulting displacement $\Delta X^{*}$ parallel to the intersection line with respect to the distance $Y^{*}$ from this line. The estimated displacement profiles are compared to an error function in order to estimate the width of the response functions:

$\Delta X(Y)=\frac{\Delta X_{0}}{2} \cdot\left(\operatorname{erf}\left[\frac{8 \cdot Y}{\mathrm{SRW}}\right]+1\right)$

where SRW is the width that covers $\operatorname{erf}(\sqrt{2}) \approx 95 \%$ of the total step height and $\Delta X_{0}$ is the step height. Figure 11 shows the fitted width as a function of the simulated particle image diameter.



Fig. 9 Response to a step-like displacement profile for different digital particle image sizes computed with single-pixel ensemble-correlation 
The lower limit of the fit function shown in Fig. 11 is at 1.84 px. This means that even the smallest particle images (1 pixel for DPIV) result in a SRW of $1.84 \mathrm{px}$. Consequently, it can be concluded from the present analysis that the best possible resolution that can be achieved in DPIV is around $1.84 \mathrm{px}$ instead of a single pixel. Furthermore, it is interesting to note that for large particle images, the resolution (defined by the SRW) is proportional to the particle image diameter. This result is consistent with the assumptions of Adrian (1997).

\subsection{Particle tracking velocimetry: resolution limit}

In the early days of DPIV, the image quality was often rather low due to low laser power and noisy due to less sensitive cameras. With correlation-based methods, the particle image displacement could be estimated very robustly compared to tracking techniques, which are very sensitive to noise. Today, high-quality images can be acquired and the center of particle images can be determined very precisely for a large range of particle image diameters and seeding concentrations by using sophisticated tracking approaches as shown by Keane et al. (1995), Ohmi and Li (2000). In principle, the accuracy is rather independent of the particle image diameter and thus not affected by the magnification as long as the particle images do not overlap. So the question arises whether digital particle tracking velocimetry (PTV) can further increase the resolution of the flow measurement? Although the statistical PTV approaches developed by Ohmi and $\mathrm{Li}$ (2000) can cope with large seeding concentrations up to 0.12 particles per pixel, the seeding concentration should be lowered to allow for a reliable detection of corresponding particle pairs. With higher seeding concentration, random errors increase due to particle image overlapping. Using a simple nearest neighbor approach works well, as long as the mean particle image spacing $\Delta \mathbf{X}_{P}$ is considerably larger than the particle image displacement $\Delta \mathbf{X}$ between two images. Almost $100 \%$ valid links between particles for synthetic particle images were achieved by Maas et al. (1993) with $\Delta \mathbf{X}_{P}=5 \Delta \mathbf{X}$.

The estimated displacement profiles using a simple nearest neighbor approach are shown in Fig. 10 for different particle images sizes ranging from 3 to 20 pixels. To allow for a better visibility, only every 1,000th data point is shown. As can be seen from the figure, the constant level of the displacement $\Delta X^{*}$ in both regions could be clearly captured. For all particle image sizes, the position of the steep change in displacement is well captured and no change in the step size can be observed by eye, meaning that the resolution is independent of the particle image size. However, it can also be noted that the scatter of the displacement estimation, and thus the uncertainty, is larger for



Fig. 10 Response to a step-like displacement profile for different digital particle image sizes computed with PTV algorithms

particle image diameters of 3 and 20 pixel in comparison with 5 and 10 . However, this scatter is related to the particle detection method used. Nobach et al. (2005) investigated different subpixel interpolation schemes for the detection of the particle center and concluded that most higher order schemes and especially a Gaussian interpolation give reliable results when the particle image diameter is larger than 2.5 pixels. For the investigation here, a wavelet-based algorithm was used (Cierpka et al. 2010), since this algorithm works much faster than a Gaussian fit and gives reliable results in a wide range of particle image sizes. Even if the intensity distribution of the particle images does not resembles a Gaussian nicely, adequate detection algorithms can be found and the rms error of the detection of the center is the only limitation for the spatial resolution for PTV. In microfluidics, another strong benefit of tracking algorithms is that the measurement error due to the depth of correlation can be avoided (Cierpka and Kähler 2011).

\section{Guidelines and recommendations}

Figure 11 summarizes the results of the response to the simulated step-like displacement profile. Using windowcorrelation, the step response shows the known dependence on the window size, but also on the digital particle image diameter as can be seen on the green curve in Fig. 11 exemplarily for a $16 \times 16 \mathrm{px}$ interrogation window. It is well known that an additional error in the velocity estimation is present due to truncated particle images. The use of weighting functions and the removal of these truncated particle images can decrease this error significantly as shown by Nogueira et al. (2001) and Liao and Cowen 




Fig. 11 Step response width of the estimated displacement with respect to the digital particle image diameter

(2005). However, reducing the interrogation window size, only few and most likely truncated particle images are present, and thus, these techniques do not improve the results any further. If the digital particle image diameter increases to approximately one-quarter of the interrogation window size, a significant influence from particles, which were partly captured in the interrogation window, is visible and the SRW increases drastically. For a particle image size of 10 pixels, which can easily happen by increasing the magnification, the SRW increases to almost 23 pixels, for instance.

Also, in the case of single-pixel ensemble-correlation, the step response indicates a dependence on the digital particle image diameter $D$. By using a curve fitting, the following relationship:

$\operatorname{SRW}(D)>1.84 \mathrm{px} \cdot \exp (-0.41 \cdot D)+0.742 \cdot D$.

limits the resolution to about 1.84 px. In the case of PTV, the SRW does not depend on the digital particle image diameter but on the error in the determination of the mean position of the particle images corresponding to a particle image pair. Using high-quality images with a good signalto-noise ratio (SNR $=100$ for the synthetic images), the determination of the digital particle image center is accurate up to $2 / 100$ of a pixel for digital particle image diameters $D>3$ pixel. Thus, the SRW for $D>3$ pixel is always below 0.05 pixel using PTV.

The field of view, with respect to the total sensor size $(L \cdot S)$, is shown in the upper plot of Fig. 12. A magnification of $M=1$, combined with a pixel size of $S=9 \mu \mathrm{m} /$ px and a sensor size of $L=4,008$ px (PCO. 4000), results in a field of view width of $36 \mathrm{~mm}$.
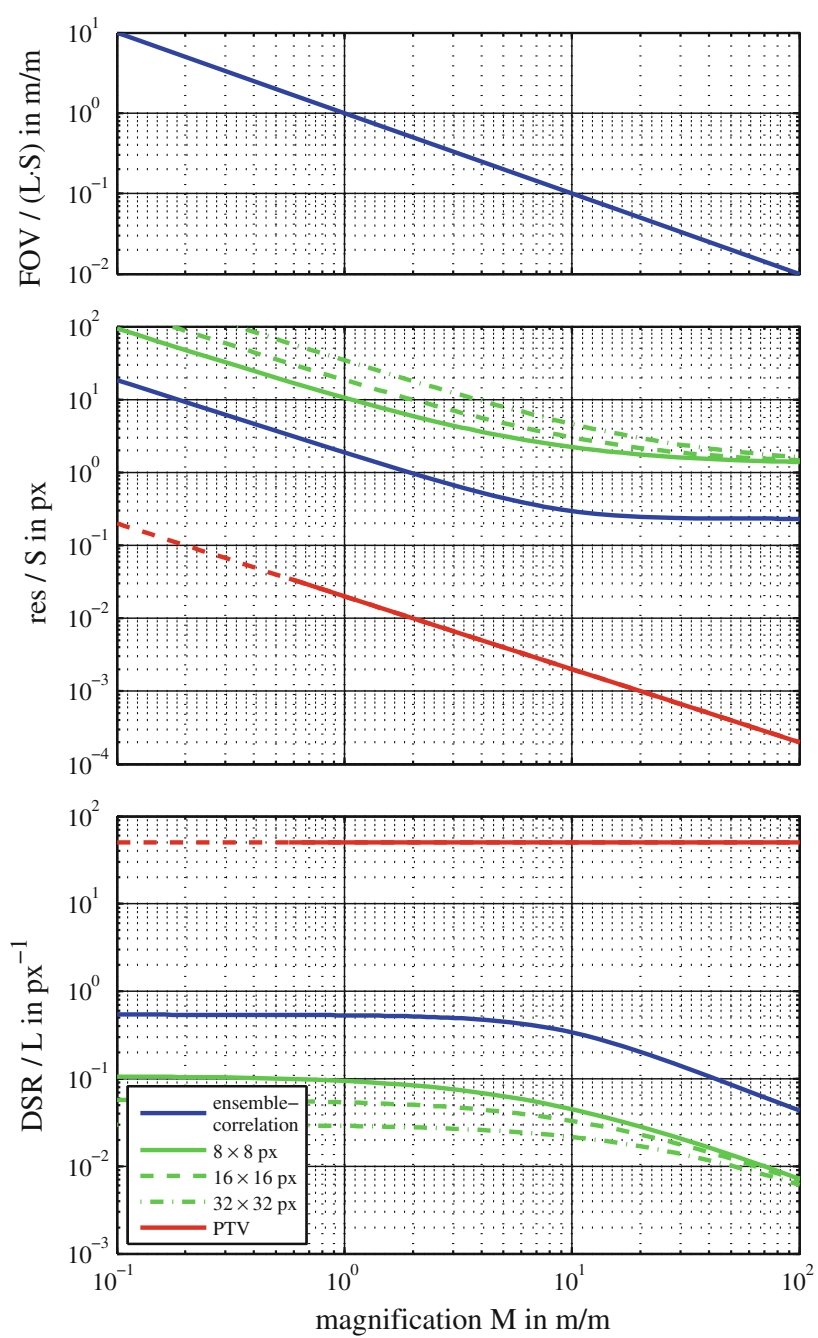

Fig. 12 Field of view (top), limits of spatial resolution (middle), and dynamic spatial range (bottom) as functions of optical magnification for $d_{\tau}=1 \mu \mathrm{m}$ and $f_{\#}=2$

\subsection{Resolution}

Combining the relationship between SRW, particle image diameter, and optical magnification allows for the determination of the resolution res on the measurement plane in physical space:

$\operatorname{res}(M)=\operatorname{SRW}(D(M)) \cdot \frac{S}{M}$

The normalized resolution is shown in the middle plot of Fig. 12 for window-correlation, single-pixel ensemblecorrelation, and PTV. For the two correlation-based methods, the graph shows the lower limit of the resolution (distance of independent vectors) determined by the SRW from Fig. 11 and the particle image size defined in Eq. 3. The resolution limit in the case of windowcorrelation is shown for three different interrogation window sizes (green solid lines) and is approximately one 
order of magnitude above the ensemble-correlation. For low magnifications and sufficient particle image density (5-10 particle images per interrogation window), the solid green lines are also valid for instantaneous displacement vector estimation. The distance between independent vectors becomes larger in case of strong lens aberrations, as they occur for large working distances, as well as in the case of out-of-focus particles or larger f-numbers.

In order to estimate the resolution limit, res, from Fig. 12, the pixel size of the camera sensor must be taken into account. The results show that for a pixel size of $9 \mu \mathrm{m}$, for example, the resolution is limited to res $>0.3 \times 9 \mu \mathrm{m}$ in case of ensemble-correlation (solid blue line), which is reached with a magnification of 10 or higher. For low magnifications like $M=0.1$, for instance, the resolution limit lies at around $200 \mu \mathrm{m}$ (for single-pixel ensemblecorrelation).

For PTV, the distance of independent mean vectors is theoretically not limited. Since the resolution is independent on the particle image diameter, using higher magnification would increase the resolution of the measurement even when particle images are very large. Especially for microscopic DPIV applications at large magnification, PTV is the only well-suited approach from the authors' point of view.

\subsection{Dynamic spatial range}

Combining the field of view and the resolution allows for the estimation of the dynamic spatial range (DSR) by following the work of Adrian (1997):

$\mathrm{DSR}=\mathrm{FOV} / \mathrm{res}$.

This relation is plotted in the lower chart of Fig. 12, normalized by the sensor size (number of pixel). The results show that in case of single-pixel ensemble-correlation (blue line), a PCO.4000 camera with $L=4,008$ would lead to a maximum dynamic spatial range of $\mathrm{DSR} \approx 0.5 \cdot 4,008 \approx$ 2, 000 for a magnification lower than $M=2$, for example. Since in case of window-correlation, the distance of independent vectors is larger than for single-pixel ensemblecorrelation, the dynamic spatial range is smaller for this evaluation method.

For very low magnifications $(M \ll 1)$, both correlationbased methods show a constant DSR value. This is due to the fact that the particle images reaches their minimum size of 1 pixel. Thus, working in this region of very small magnification might also cause peak locking.

The dynamic spatial range of PTV is in principle independent on the optical magnification. However, optimal conditions for PTV are a low seeding concentration and fairly large particle images $(D \approx 3 \ldots 15 \mathrm{px})$. Thus, PTV requires, in general, magnifications of $M>0.5$.

\section{Summary}

The impact of a localized flow gradient (a step function) on the resolution limit of DPIV was examined in detail using window-correlation, single-pixel ensemble-correlation, and particle tracking image analysis methods.

It was shown that for single-pixel ensemble-correlation, the resolution is dependent on the particle image diameter, whereas in the case of state-of-the-art window-correlation analysis, the spatial resolution depends on the interrogation window size. For PTV, on the other hand, the particle image diameter does not limit the spatial resolution.

The particle image diameter has a lower limit, which is determined by the particle size, the pixel size of the camera sensor, the f-number of the imaging system, and optical aberrations. Experimental particle images values for varying magnifications are always larger than the theoretical limits derived. In case of a large working distance $(1 \mathrm{~m})$, the diameter is enlarged by almost one order of magnitude due to lens aberrations.

For the two correlation-based methods, the analysis shows that the resolution cannot be reduced below a certain point, which is defined by the particle size $d_{\mathrm{p}}$, the pixel size $S$, and the f-number. Magnifications larger than $M>10$ do not seem to further improve the resolution for typical experimental conditions $\left(f_{\#}=2\right.$ and $\left.d_{\mathrm{p}}=1 \mu \mathrm{m}\right)$ since the particle images grow linearly with the magnification. Furthermore, the dynamic spatial range decreases with increasing magnification.

In order to achieve results with the best possible spatial resolution and dynamic spatial range by means of correlation methods, it is recommended to: (1) use the smallest possible working distance (by taking perspective errors into account), (2) select a high-quality objective lens with low f-number, (3) use a camera with small pixel size and large sensor, and (4) acquire a sufficient amount of DPIV recordings for single-pixel or sum-of-correlation evaluation. The best resolution in terms of independent velocity vectors is nevertheless limited for correlation-based methods. For large magnifications, the resolution saturates at a value that depends on the particle size, the pixel size of the camera, the f-number of the objective lens, and lens aberrations. PTV evaluation does not have the same limits, it can achieve better resolution. However, it requires a high signal-to-noise ratio and a relatively low seeding concentration to avoid overlapping particle images.

Since velocity vectors can be determined for each particle image pair in a certain region, by using a PTV algorithm the whole velocity probability density function (pdf) is available, in principle, and higher order moments can be estimated directly. It is also possible to estimate the Reynolds stresses from the pdf of the velocity in the case of ensemble-correlation or sum-of-correlation. Thereby, the 
shape of the correlation function and the shape of the autocorrelation function need to be analyzed as outlined in Scharnowski et al. (2011).

If the temporal development of the flow is of interest, window-correlation-based methods must be used. In this case, however, the resolution is diminished when estimating mean velocities. However, the resolution is limited by the same parameters with the addition of the seeding concentration. In this case, the particle image size and density determine the interrogation window size that directly influences the resolution. At least 6-8 particle images are required per interrogation window to limit the number of spurious vectors to an acceptable level. For a digital particle image diameter of $D=3 \mathrm{px}$ and a seeding concentration of $25 \%$, the interrogation window size should be larger than $14 \times 14 \mathrm{px}$. Since the resolution of correlation-based methods is limited by the particle image diameter, it should be possible, in principle, to increase the resolution with an appropriate preprocessing method that reduces the particle image size.

Acknowledgments The financial support from the German Research Foundation (DFG) in the framework of the Sonderforschungsbereich Transregio 40 and the Individual Grants Programme KA $1808 / 8$ is gratefully acknowledged by the authors. Technical language revisions by R. Segura and fruitful discussions with R. Hain are also appreciated.

Open Access This article is distributed under the terms of the Creative Commons Attribution License which permits any use, distribution, and reproduction in any medium, provided the original author(s) and the source are credited.

\section{References}

Adrian RJ (1997) Dynamic ranges of velocity and spatial resolution of particle image velocimetry. Meas Sci Technol 8:1393. doi: 10.1088/0957-0233/8/12/003

Adrian RJ, Westerweel J (2010) Particle image velocimetry. Cambridge University Press, Cambridge

Billy F, David L, Pineau G (2004) Single pixel resolution correlation applied to unsteady flow measurements. Meas Sci Technol 15:1039-1045. doi:10.1088/0957-0233/15/6/002

Bitter M, Scharnowski S, Hain R, Kähler CJ (2011) High-repetitionrate PIV investigations on a generic rocket model in sub- and supersonic flows. Exp Fluids 50:1019-1030. doi:10.1007/s00348010-0988-8

Cierpka C, Kähler CJ (2011) Particle imaging techniques for volumetric three-component (3D3C) velocity measurements in microfluidics. J Vis 15:1-31. doi:10.1007/s12650-011-0107-9 open access

Cierpka C, Segura R, Hain R, Kähler CJ (2010) A simple single camera $3 C 3 \mathrm{D}$ velocity measurement technique without errors due to depth of correlation and spatial averaging for microfluidics. Meas Sci Technol 21:045401. doi:10.1088/0957-0233/21/4/045401

Hain R, Kähler CJ (2007) Fundamentals of multiframe particle image velocimetry (PIV). Exp Fluids 42:575-587. doi:10.1007/s00348007-0266-6
Hain R, Kähler CJ, Tropea C (2007) Comparison of CCD, CMOS and intensified cameras. Exp Fluids 42:403-411. doi:10.1007/ s00348-006-0247-1

Kähler CJ (2004) The significance of coherent flow structures for the turbulent mixing in wall-bounded flows. $\mathrm{PhD}$ thesis, GeorgAugust-Universität zu Göttingen. http://webdoc.sub.gwdg.de/ diss/2004/kaehler/kaehler.pdf

Kähler CJ, Kompenhans J (2000) Fundamentals of multiple plane stereo particle image velocimetry. Exp Fluids 29:S070-S077. doi:10.1007/s003480070009

Kähler CJ, Sammler B, Kompenhans J (2002) Generation and control of particle size distributions for optical velocity measurement techniques in fluid mechanics. Exp Fluids 33:736-742. doi: 10.1007/s00348-002-0492-x

Kähler CJ, Scholz U (2006) Transonic jet analysis using long-distance micro PIV. 12th international symposium on flow visualization, Göttingen, Germany, Sep 10-14

Kähler CJ, Scholz U, Ortmanns J (2006) Wall-shear-stress and nearwall turbulence measurements up to single pixel resolution by means of long-distance micro-PIV. Exp Fluids 41:327-341. doi: 10.1007/s00348-006-0167-0

Keane RD, Adrian RJ (1990) Optimization of particle image velocimeters. Part I: Double pulsed systems. Meas Sci Technol 1(11):1202-1215. doi:10.1088/0957-0233/1/11/013

Keane RD, Adrian RJ, Zhang Y (1995) Super-resolution particle image velocimetry. Meas Sci Technol 6:754-768. doi:10.1088/ 0957-0233/6/6/013

Liao Q, Cowen EA (2005) An efficient anti-aliasing spectral continuous window shifting technique for PIV. Exp Fluids 38:197-208. doi:10.1007/s00348-004-0899-7

Maas H, Gruen A, Papantoniou D (1993) Particle tracking velocimetry in 3-dimensional flows. 1. Photogrammetric determination of particle coordinates. Exp Fluids 15:133-146. doi: 10.1007/BF00190953

Meinhart CD, Wereley ST, Santiago JG (2000) A PIV algorithm for estimating time-averaged velocity fields. J Fluids Eng 122:285-289. doi:10.1115/1.483256

Melling A (1997) Tracer particles and seeding for particle image velocimetry. Meas Sci Technol 8/12:1406-1416. doi:10.1088/09570233/8/12/005

Nobach H, Damaschke N, Tropea C (2005) High-precision sub-pixel interpolation in particle image velocimetry image processing. Exp Fluids 39:299-304. doi:10.1007/s00348-005-0999-z

Nogueira J, Lecuona A, Rodriguez PA (2001) Identification of a new source of peak locking, analysis and its removal in conventional and super-resolution PIV techniques. Exp Fluids 30:309-316. doi: $10.1007 / \mathrm{s} 003480000179$

Nogueira J, Lecuona A, Rodriguez PA (2005) Limits on the resolution of correlation PIV iterative methods. Fundamentals. Exp Fluids 39:305-313. doi:10.1007/s00348-005-1016-2

Ohmi K, Li HY (2000) Particle tracking velocimetry with new algorithms. Meas Sci Technol 11:603-616. doi:10.1088/09570233/11/6/303

Olsen MG, Adrian RJ (2000) Out-of-focus effects on particle image visibility and correlation in microscopic particle image velocimetry. Exp Fluids 29:S166-S174. doi:10.1007/s003480070018

Raffel M, Willert CE, Wereley ST, Kompenhans J (2007) Particle image velocimetry: a practical guide. Springer, Berlin

Rossi M, Segura R, Cierpka C, Kähler CJ (2011) On the effect of particle image intensity and image preprocessing on depth of correlation in micro-PIV. Exp Fluids. doi:10.1007/s00348-011-1194-Z

Scarano F (2001) Iterative image deformation methods in PIV. Meas Sci Technol 13:R1-R19

Scharnowski S, Hain R, Kähler CJ (2011) Reynolds stress estimation up to single-pixel resolution using PIV-measurements. Exp Fluids. doi:10.1007/s00348-011-1184-1 (in print) 
Scholz U, Kähler CJ (2006) Dynamics of flow structures on heaving and pitching airfoils. In: 13th international symposium on applications of laser techniques to fluid mechanics, Lisbon, Portugal

Stanislas M, Okamoto K, Kähler CJ (2003) Main results of the first international PIV challenge. Meas Sci Technol 14:R63-R89. doi: 10.1007/s00348-005-0951-2

Stanislas M, Okamoto K, Kähler CJ, Westerweel J (2005) Main results of the second international PIV challenge. Exp Fluids 39:170-191. doi:10.1007/s00348-005-0951-2

Stanislas M, Okamoto K, Kähler CJ, Westerweel J, Scarano F (2008) Main results of the third international PIV Challenge. Exp Fluids 45:27-71. doi:10.1007/s00348-008-0462-z
Stitou A, Riethmuller ML (2001) Extension of PIV to super resolution using PTV. Meas Sci Technol 12:1398-1403

Wernet JH, Wernet MP (1994) Stabilized alumina/ethanol colloidal dispersion for seeding high temperature air flows. In: Proceedings of the ASME symposium on laser anemometry: advances and applications, Lake Tahoe, NV, 19-23 June

Westerweel J (2008) On velocity gradients in PIV interrogation. Exp Fluids 44(5):831-842. doi:10.1007/s00348-007-0439-3

Westerweel J, Geelhoed PF, Lindken R (2004) Single-pixel resolution ensemble correlation for micro-PIV applications. Exp Fluids 37:375-384. doi:10.1007/s00348-004-0826-y

Willert C (1997) Stereoscopic particle image velocimetry for application in wind tunnel flows. Meas Sci Technol 8:1465-1479 